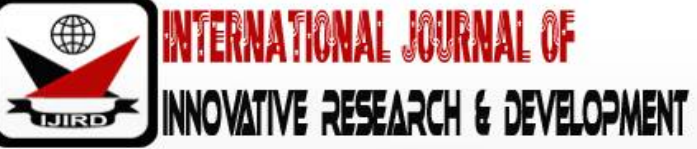

ISSN 2278 - 0211 (Online)

\section{Arduino Based Smart Energy Saving System in Ugandan Public Universities: A Case Study of Muni University, Uganda}

Lawrence Nkamwesiga
Lecturer, Department of Computer and Information Science, Muni University, Uganda,
Julius Junior Kazibwe
Graduate Student, Department of Computer and Information Science, Muni University, Uganda
Paul Male
Graduate Student, Department of Computer and Information Science, Muni University, Uganda

\begin{abstract}
:
Abstract: The application of internet of things in the real world offers numerous benefits including smart homes and offices, a technology that does not only save energy but also saves money. Office automation is becoming popular due to its numerous benefits as applied in the world of internet of things. The specific objectives of the study were: to assess the current energy usage, identify current energy saving measures, and design and implement a smart energy saving system for Muni University. System requirements were collected from the respondents who were staff of Muni University. The study considered 2 respondents from estates office, 2 from procurement, 1 from each of the three departments of education, Computer and Information Science, and Nursing Sciences. User requirements were gathered a qualitative protocol using focus group discuss and thematic analysis technique was employed. Heterogeneous home automation systems and technologies were considered in review with central controller-based Arduino, sensors, web based, email, Bluetooth, mobile, SMS, ZigBee, Dual Tone Multi Frequency, and cloud based. The study design utilized the Global system for mobile communication technology as a user interface using SMS based communication with Arduino as the central controller. The system supports internet of things concept that can be applied in saving electrical energy usage in public places including public universities. The study further recommends the Ugandan government to advocate for Arduino Based Smart Energy Saving System that can reduce electrical energy expenditure in Ugandan public universities Muni University inclusive.
\end{abstract}

Keywords: Home-automation, intelligence, Arduino, microcontroller, global system for mobile communication, smart energy, Muni University Uganda

\section{Introduction}

Automation is a technique of controlling a process by electronic devices with reducing human involvement to a minimum. The fundamental of building an automation system for an office or home is increasing day-by-day with numerous benefits. Industrialist and researchers are working to build efficient and affordability automatic systems to monitor and control different machines like lights, fans, air conditioners based on the requirements (Pan, et al., 2015). The automation method makes effective use of electrical energy that eventually results in saving costs while reducing energy waste. This project is based on the concept of internet of things (IoT) that allow objects including people and other devices interconnected over the internet anytime and anywhere using any network and service to exchange data and information. Automation is another important application of IoT technologies. IoT can be applied in setting up smart homes and offices (Pan, et al., 2015). It can be used for the monitoring of energy consumption and controlling the environment in buildings, schools, offices and museums by using different types of sensors and actuators that control lights, temperature, and humidity (Minoli, et al., 2017).In nest apartments, they have been using WIFI home automation system to save energy and controlling electrical appliances. The tenants access a web-based platform which allows them to control the electrical appliances in their rooms. But this system can be hacked into. However, in Nigeria, United kingdoms, India and china they use GSM home automation system to save energy and control electrical appliances. The system is much safer and secure than WIFI system because it cannot be hacked into.

Currently, Muni University controls electrical appliances using three different modes: Manual system which is used in buildings, the timer automation system which is used for garden lights.it turns on and off after every twelve hours, the light sensor system which is used on security lights. With low levels of light intensity, it turns on the security lights. The current does not support remote control of the electrical appliances via the phone. The proposed Arduino Smart Energy Saving Systems offers flexibility and remote sensing capabilities. 


\subsection{Statement of the Problem}

Manual means to save electric energy continue to dominate as a means of saving costs in Ugandan public universities including Muni University although they are inconsistent (E Source Companies LLC, 2003).Currently, Muni University uses light intensity sensors as a way of controlling only security lighting system which are disadvantaged when there is low light intensity during the day the security lights switches on themselves hence causing additional energy costs unnecessary. The current control system lacks remoteness and hence if a user forgets the lights on, there is no way the estate manage can check whether the lights are on or off. As a result, this inflicts a heavy budget burden on the Ugandan government in running public Universities including Muni University and the parents as well. A report produced by the University World News (2019) indicated that in Ghana, the highest tuition cost was due to utilities such as electricity. Furthermore, Muni University pays two million four hundred shillings per month on electricity which is expected to go beyond as University needs increase. This causes a burden cost incurred by students as well as the government a burden to tuition This project therefore proposes an Arduino Based Smart Energy Saving System for Muni University that also adopted by other Ugandan Public Universities to reduce utility bills

\subsection{Objectives}

\subsubsection{General Objectives}

The main of objective is to design and implement Arduino-GSM abased electric energy saving system for Muni University that will minimize utility bills.

\subsubsection{Specific Objectives}

To assess the current electric energy usage at Muni University

- Identify current electric energy saving measures at Muni University

- Design and Implement a smart energy saving system for Muni University

\subsection{Research Questions}

- What is the current electric energy usage at Muni University?

- What measures are used to save energy at Muni University?

- How can a smart energy saving system for Muni University be designed and implemented?

\subsection{Scope of the Study}

The project intended to design the electric energy saving system for Muni University that switches off electrical appliances remotely using Arduino and Global system for communication (GSM) technology. The project started on May 30, 2017 and ended on April 20, 2018. The whole project template costed UGX 3000000 (US \$811).

\subsection{Significance of the Project}

Arduino system enables the custodian to control electrical appliances using his mobile phone which will reduce on power consumption hence reduction on heavy electricity bills.

\section{Literature Review}

\subsection{Introduction}

There have been numerous researches works in the literature where the issue of automating home has been addressed using different communication technologies. Previous works can be categorized based on communication medium used as: internet, DTMF signaling, GSM network, Bluetooth, and ZigBee technology. In what follows, a review of home automation research using these communication mediums is hereby presented.

\subsection{Review of Related Literature}

\subsubsection{Home Automation Using Bluetooth}

Bluetooth is a wireless technology for transferring data between two devices that are in close proximity; it is most suitable for short timed communications. Bluetooth communication technology does not require line of sight between the two communicating nodes. Thus, making it a viable option for home appliance control over the traditional remotecontrolZhao and Zhaohui (2008) developed "PICONET", which basically is a network of Bluetooth devices. The authors made use of a master-slave connection between several devices. The control or central Bluetooth module is attached to a microcontroller chip which serves as the master device while the slaves are the modules attached to the various appliances. An android phone was used as the mobile unit and commands were issued via the Bluetooth application on it. The microcontroller was set in a polling status and constantly checks any input command every 500 millisecond from the Android phone application.

The use of master-slave structure ensured that existing structures are not tampered with and scalability becomes easy. However, control from remote location and cost of implementing this on a large scale is still a huge limitation. A low cost, flexible and secure cell phone-based home automation system was discussed (Prantosh, et al., 2017). The home appliances were connected through relay to the I/O port of a stand-alone Arduino board for the control of home 
environment devices. The system design was enhanced with the incorporation of security assistance using password technique to prevent unauthorized user to the smart home control system.

Therefore, the mobile phone operation was script in python programming language which allows it to function on any platform of Symbian OS. The system is capable of switching ON/ OFF appliances without the need for internet connectivity. The performance range in a concrete building was reported to be greater than $50 \mathrm{~m}$, and in an open area to be $100 \mathrm{~m}$. The Bluetooth based smart home design in which an android application was used as the control interface was presented (Bala, et al.,2012). The application allowed the user to control devices by simply pushing ON/ OFF buttons on the application interface. On activation, the application creates a session with the Bluetooth module at the control unit, formats the commands as required and sends it via the mobile phones Bluetooth interface. On reception of this commands, the Microcontroller processes the data and sends necessary control signals to the connected relays which in turn switches the state of the appliances as required. The system is user friendly and more devices can easily be integrated. However, the limited range of Bluetooth still remains a major drawback as control from remote locations is impossible

\subsubsection{Home Automation Using ZigBee}

Some other authors have considered using the ZigBee wireless technology for home automation system. ZigBee is a radio frequency (RF) communications standard based on IEEE 802.15.4.A ZigBee-based home automation system was put forward to address the shortcoming of connectivity and interoperability of home appliances (Manohar \& Kuman. 2015). ZigBee wireless technology and Wi-Fi network for home automation was integrated through a gateway, virtual home system for control and monitoring home devices using remote control system. The implementation of home gateway was to provide network interoperability, flexibility of user interface, simple connectivity and to permit remote access to the home system environment. In addition, the virtual gateway was implemented to cater for security and safety requirements. The system was proposed to cater for four home appliances like switching of light, valve warmer, safety sensor and remote control.

A Security control and monitoring system based on ZigBee technology was developed (Khusvinder, et al., 2009). The system comprises of ZigBee modules and ZigBee end devices. The system was designed to control electrical doors and gas system via a switching circuit which is also equipped with a ZigBee node. Whenever intrusion is detected, the home owner is notified via SMS. The owner can send a command to shut all doors and activate the gas system depending on the need. The proposed system has a basic advantage over Bluetooth technology-based systems in that it has a far better range and generally ZigBee modules consume less power compared to Bluetooth modules. However, control is still limited by range. A voice control system was developed for a ZigBee based home automation system (Jinsoo \& Park, 2010; Barak, et al., 2013) while a wireless smart home security system based on ZigBee and GPRS technology wacame later(Motshed, et al., 2015). It consisted of a host control system and other sub functional modules. The host control system was made up of the GPRS module, ZigBee module and PIR sensor. The Sub functional modules consist of the data acquisition module, centralized switch and ZigBee module. It utilized the master-slave structure kind of communication between the centralized ZigBee module and the sub functional modules. The host sends command messages via GPRS mobile phone to the master slave or host control center which drives the control of appliances connected to it. Using this concept an energy efficient Design of Home Automation System (HAS) was proposed (Carelin \& Raglend, 2011). The system consists of network of sensors and appliance actuators to get information and control house environment respectively. An Arduino microcontroller was used as a central controller for communicating with an Android application and user interface.

\subsubsection{Home Automation Using GSM Network}

Short Message Service (SMS) allows text messages to be sent and received using mobile phones. The text can comprise of word, numbers, or an alphanumeric combination. Empirical literature indicates that by 2010, the application that was widely used was SMS data, with an estimated 3.5 billion users active (about $80 \%$ of all mobile phone subscribers) (Amponsah, et al.,2018). The ease of use and popularity of this communication system explains why it is widely deployed in smart home development.

HAS for monitoring and controlling household appliances in remote locations can be achieved through various communications technologies such as wireless LAN technology, dial-up modems, Bluetooth, Zig-Bee, satellite communication and cellular networks. Among the options of these technologies, cellular network has attracted attention of researchers due its wide coverage area, cost effectiveness compares to other mobile communications and it is a highly secured network. The cellular network mostly employed is the GSM (Global System for Mobile communication) standard. An empirical study indicates that a design and implementation of a machine-2-machine (M2M) system using GSM network was proposed to support service automation (Alkar \& Buhur 2005). Alkar and Buhur, further stated that the system can be implemented using many technologies such as Dual Tone Multi Frequency (DTMF), Short Message Services (SMS), and General Packet Radio Services (GPRS). In this project Short Message Services (SMS) was used. The designer is to choose the most suitable technology for any particular application. Yan and Shi (2013) proposed the design of a basic smart home system which allows users to control home appliances via text messaging over a given GSM network. The design was targeted at providing a simple and easy to control smart home system that is very affordable. The mobile unit (user) sends control commands to the mobile device stationed at the control center. The microcontroller then extracts this command from the mobile device, processes the commands and sends control signals to the relay drivers which in turn drives the relays thereby switching the connected devices ON/ OFF. The user then gets a feedback message showing the commands executed and current state of the devices. The system has the advantage of simplicity and ease of implementation but the drawbacks are that the system is not robust and one might encounter much difficulty in integrating new devices into the system. In addition, frequent use of system indicates higher cost over time. Another approach was proposed (Sougata, et 
al.,2014) to developed a framework for home automation using J2EE/J2ME platform. In this system, the control of household is done with the aid of mobile phones. The paper analyses the design plan of both the server terminal and the mobile phone user. Unified Model Language (UML) was also used in the modelling of HAS. However, this approach requires internet service and also depends on the battery of the mobile phone to be able to control the devices. Therefore, the system may not be reliable in the absence of internet and when the battery of the mobile phone is drained out (Statt, 2016). In this project the concept of controlling household appliances using cell phone with the aid of GSM technology was developed. The system uses SMS to control household appliances from far distance. It is more adaptable and cost effective and also provides ubiquitous access especially where internet facilities are not available. Other similar works have also been presented (Siwen \& Yunhong, 2004; Teymourzadeh, 2013; Tulijappa, 2009; Vini, M, \& Reddy, 2012).

\subsubsection{Home Automation Using DTMF Signaling}

Dual-tone multi-frequency signaling (DTMF) is an in-band telecommunication signaling system using the voicefrequency band over telephone lines between telephone equipment, other communications devices, and switching centers (Afshan, et al., 2014). The tones produced when dialing on the keypad of mobile phone could be used to represent the digits since a separate tone is used for each digit(Alheraish, 2004). It makes use of the DTMF, two mobile phones, DTMF decoder, and an atmega8 microcontroller. One mobile phone was used as the remote device via which the user can send control commands, while the second mobile phone as the receiving device. This approach is expensive as it requires two phones, not user friendly; users will need to memorize control commands to operate a given device. In addition, a phone call must always be established with the mobile phone at home which introduces delay to the system. In Uganda, UMEME (2018)resented a home automation system using DTMF with a noise reduction features using Goertzel DFT estimation to eliminate the noise in the system. Empirical literature indicates that similar works were done using the DTMF approach was presented.

\subsubsection{Internet-Based Home Automation}

The internet became a common place in the twentieth century and the basic advantage it holds over all other forms of wireless communication is that it is unlimited by distance or location and it is universal. The internet has formed the backbone for a new trend in home automation popularly referred to as the Internet of Things (IoT). Since internet connectivity is becoming everywhere which also help in driving the IoT technology, an IP-based home automation system was proposed (Shaker \& Fahtha, 2009). In order to simplify the cost and complexity of IP-based system, the authors developed a hardware/ software framework called IPAcBox which allows home/ office automation systems to be accessed and controlled through a direct connection right out of the box. The system also works in the event of dynamic IP configurations. However, the system is expensive to implement compare to other GSM based approach.Anwaarullah and Altaf (2013)came up with a ubiquitous COAP-based home automation system using time synchronization for the development of low power sensor modules with smart home control system. COAP protocol was standardized recently for the sensor network application to operate between M2M in the smart home appliances. The authors proposed that COAP scheme can be applied to both IP-based and non-IP-based home automation gadgets. This lightweight Constrained Application Protocol is a web-based application-level protocol that supports IEEE 802.15.4 low level standard for sensor networks and supports (RESTful) architecture. The time synchronization scheme presented in their paper was aimed at minimizing both resource usage and network overhead that exist on the sensor nodes and home gateway, in order to achieve better time accuracy in comparison with existing design.

An Internet Based Wireless Home Automation System for Multifunctional Devices was discussed (Mohd \& Veda, 2013). The aim of the paper was to design and implement a central point control home automation system, low-cost, flexible and secure internet-based system. The authors suggested a framework for a communication protocol that operates between several devices available in the home automation than common switching techniques. The system connections between the different nodes (i.e., the user interface, the database and the web-based connections) were modeled in unified modeled language (UML). In the hardware system design, PIC16F877 microcontroller was used with RF wireless technology to transmit signal via MAX232 serial port to the web server. However, the system was demonstrated to control only switching of light in the home environment. An internet-based home automated system was proposed in which a smart home developed on an Arduino platform was controlled and monitored via Email over the internet (Jieming, et al., 2010; John, et al., 2010). A user-friendly HAS based on 3D virtual world was proposed (Wahab, et al., 2010). The paper proposed design of a realistic 3D view interface as an improvement on the existing user interface. In addition, a home server was used as a controller for home devices. With this 3D virtual World, a user can control and monitor home devices via a user-friendly interface that works both intuitively and realistically anywhere and anytime through the Internet. High cost and availability of internet still remains the shortcomings of the system. A review of IoT technologies, architecture, challenges, applications and future trend have been presented (Oladlmeji, Mustapha \& Lukman, 2017).

\subsubsection{Home Automation Using Multiple Technologies}

The most important factors that should influence the design of a smart home system include the scalability of the system, security, ease of integrating new appliances and devices into the existing design, ease of use or a user-friendly control interface and cost effectiveness of the overall system. In recent designs, researchers are faced with a tradeoff between most of this factor as the pursuance of one of these factors may limit the implementation of another. To implement such a system that integrates all these factors, some researchers have proposed HAS that utilizes two or more of the available wireless technologies (Piyare \& Tazil, 2011) combined both GSM and Bluetooth technology for monitoring and control of home appliances and sensors. A combination of ZigBee and GSM technology was proposed (Oladlmeji, 
Mustapha \& Lukman, 2017; Motshed, et al., 2015), that combined four different technologies; Bluetooth, GSM, Wi-Fi and DTMF to achieve a low cost, flexible, and standalone of controlling the home appliances, however, the use of too many communication technologies can introduce complexity in the system thus leading to high cost of production.

\subsection{Gaps Identified In Related Studies}

Several novel approaches have been proposed in the literature for home automation system, however, there still exits some limitations ranging from high cost of implementation to complex control mechanism. To minimize the various drawbacks evident in all the a fore mentioned systems, the system finished here implements a number of features and measures to achieve a low-cost smart automation system for Muni University. And also, since the population is more on smart phones using the social media it becomes easy for them to use their mobile phone to control the appliances

\section{Methodologies}

\subsection{User Requirements}

User requirements were gathered using an interview guide instrument guided by a qualitative research protocol from 5 five departments of Muni University using quota purposive sampling technique using the respondents were purposely selected from each department as follows: 1 procurement, 2 Estates, 1 Computer and Information Science, 1 Nursing, and 1 education (Coyne, 1997).Qualitative data from the interview guide were analyzed using thematic analysis until saturation.

\subsection{Design Methods}

\subsubsection{Design Methods}

The project used incremental model as the design method where the whole requirements were divided into various builds and multiple development cycles take place here, making the life cycle a "multi-water fall" Cycles are divided up into smaller, more easily managed modules which were developed one after another. In this model, each module passes through the requirements, design, implementation and testing phases. A working version of application was produced during the first module and we had a working application version early on during the development process. Each subsequent release of the module added functionality on to the previous release. The process continued till the complete application version was achieved. The design steps used were as follows:

And these are the steps involved in the development

\subsubsection{Step 1- Planning \& Requirements}

As with any development project, the first step is going through an initial planning stage to map out the specification documents, establish software or hardware requirements, and generally prepare for the upcoming stages of the cycle. At this stage gathered the necessary requirements for the development of the application from the sample population using the different data collection methods mentioned above.

\subsubsection{Step 2-Analysis \& Design}

After completing planning, we performed an analysis to nail down the appropriate business logic, database models, and the others required at this stage in the project. We designed at this stage and also establishing the technical requirements (languages, data layers, services, etc.) that we used to meet the needs of the analysis stage.

\subsubsection{Step 3-Testing}

Once this current build iteration was coded and implemented, the next step was to go through a series of testing procedures to identify and locate any potential bugs or issues that have cropped up.

\subsubsection{Step 4-Implementation}

With the planning and analysis finished, the actual implementation and coding process began here. All planning, specification, and design documents were coded and implemented into this initial iteration of the project.

Diagram for the incremental development 


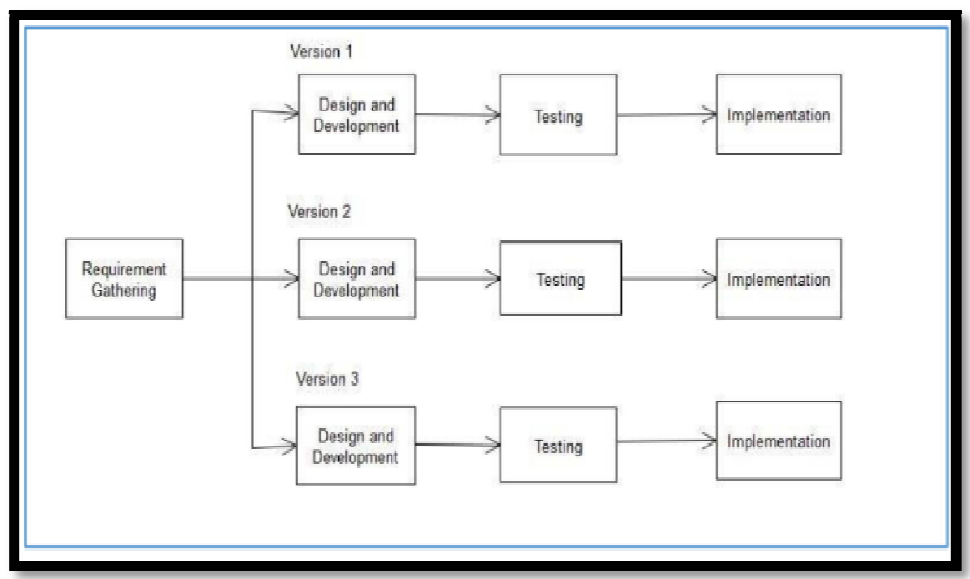

Figure 1: Incremental methodology diagram

\subsubsection{Conceptual Design}

This section describes the conceptual design of the proposed smart university energy saving system. The system block diagram is as shown in Fig. 1. Users can control the devices remotely through the GSM network using the mobile phones. The block diagram shows the connection of the GSM module, which serves as the SMS gateway, the Relay module, Arduino and LCD. A 12 V battery is used to power the Arduino microcontroller board which powers the rest of the system. The operation of the hardware solely depends on the program code running on the microcontroller chip. The program is written using the Arduino IDE. The LCD displays the various status of the system.

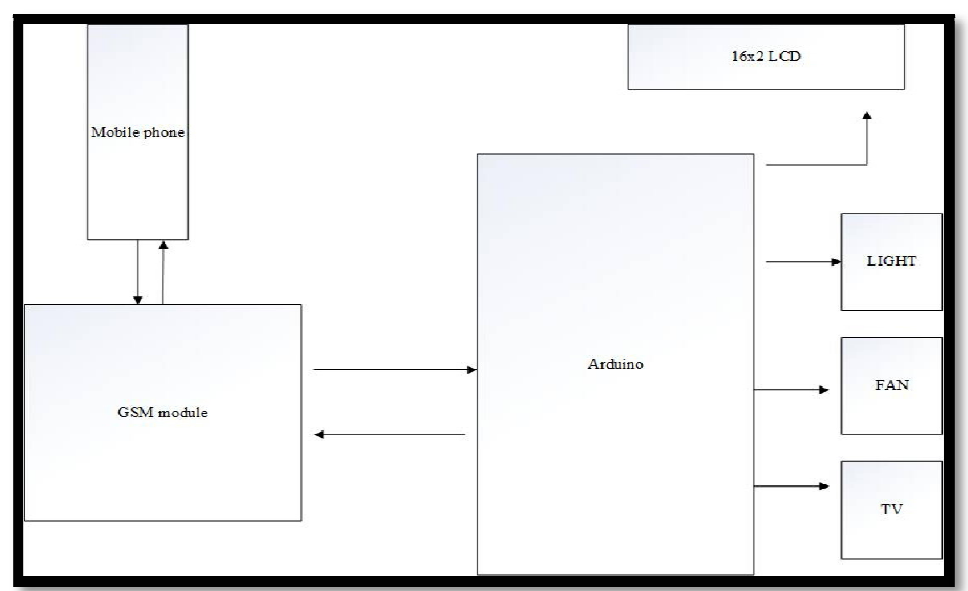

Figure 2: The Functional Blok Diagram of the Proposed System

\subsubsection{GSM Technology}

In our system, the SMS (Short Message Service) feature of GSM technology is used to achieve control from a remote location. SMS can make all communication in short. It has gained widespread application in home appliance control this is because it allows users to control home appliances from a remote location by sending a short command. However, for the purpose of flexibility, users without smart phone can also control the appliances by using SMS to send the required commands. The SMS sending and receiving functions are achieved using a SIM900A GSM module. The module is provided with a SIM (Subscriber Identity Module) which enables it to send and receive SMS. It can work with SIM from any of the available network providers. The module is configured using standard AT commands and is capable of performing operations of conventional mobile phones. The module used here also supports an extended AT command set. It has current consumption of 250mA during normal operation. It also supports Voice, Fax/ Data GPRS and integrated TCP/ IP suite and is connected to the microcontroller.

\subsection{GSM Module}

A GSM modem is a specialized type of modem which accepts a SIM card, and operates over a subscription to a mobile operator, just like a mobile phone. GSM (Global system for mobile communication) uses a process called circuit switching. This method of communication allows a path to be established between two devices. Once the two devices are connected, a constant stream of digital data is relayed. GSM networks consist of three major systems the Switching System (SS), The Base Station (BSS) and the Mobile Station (MS).

\subsubsection{The Switching System}

The Switching system is very operative system in which many crucial operations are conducted, switching systems holds five databases with in it which performs different functions. The major tasks the Switching System performs are call processing and subscriber related functions. These databases from switching systems are HLR, MSC, VLR, AUC and 
EIR. The Mobile Switch Center (MSC) in cooperation with Home Location Register (HLR) and Visitor Location Register (VLR), take care of mobile calls and routing of phone calls. Authentication Centre (AUC) is small unit which handles the security end of the system and Equipment Identity Register (EIR) is another important database which holds crucial information regarding mobile equipment. The base station system has very important role in mobile communication. BSS are basically outdoor units which consist of iron rods and are usually of high length. BSS are responsible for connecting subscribers (MS) to mobile networks. All the communication is made in Radio transmission. The Base Station System is further divided in two systems. These two systems are BTS and BSC. BTS (Base Transceiver station) handles communication using radio transmission with mobile station and BSC (Base station controller) creates physical link between subscriber (MS) and BTS, then manage and controls functions of it

\subsubsection{Mobile Station (Subscriber)}

MS consist of a mobile unit and a smart card which is also referred as a Subscriber Identity Module (SIM) card. This card fitted with the GSM Modem and gives the user more personal mobility. The equipment itself is identified by a unique number known as the International Mobile Equipment Identity (IMEI). The GSM modem used in this device is SIM 900A.The parameters and specification of our GSM modem is given below.

\begin{tabular}{|l|l|l|l|l|}
\hline \multicolumn{5}{|c|}{ GSM Modem SIM900A Parameter and Specification } \\
\hline Frequency band & Power transmission & Baud rate & Power supply & Operating temperature \\
\hline Quad band 850/900/1800/1900 & $\begin{array}{l}2 \mathrm{~W} @ 850 / 900 \mathrm{MHz} \\
1 \mathrm{~W} @ 1800 / 1900 \mathrm{MHz}\end{array}$ & 9600 & $5 \mathrm{~V}, 1.5 \mathrm{~A}$ & $-40{ }^{\circ} \mathrm{C}$ to $85{ }^{\circ} \mathrm{C}$ \\
\end{tabular}

Table 1: GSM Modem SIM900A Parameter and Specification

\subsubsection{Arduino System}

It is a microcontroller board, not fully computers. In this, written codes are simply executed without any obstacle. It is an 8-bit Atmel AVR Microcontroller which comprises of 32K and 512K of onboard flash memory, 2K of RAM, runs at 8$84 \mathrm{MHz}$ clock speeds with voltages of $2.7 \mathrm{~V}-12 \mathrm{~V}$.programming is done using $\mathrm{C}++$ and carries no operating system. The code is written in the computer and then sent through USB cable for execution. Its construction simply covers digital inputoutput pins that are between 9-54 AND 6-12 analog input pins. Its power consumption is less than 0.5 watt. Firmware code refers to the program running on the microcontroller chip. The program was written be written and debugged using $\mathrm{C}++$ language from the Arduino IDE which is a free open source software. The flowchart of the program is shown in Fig.3.

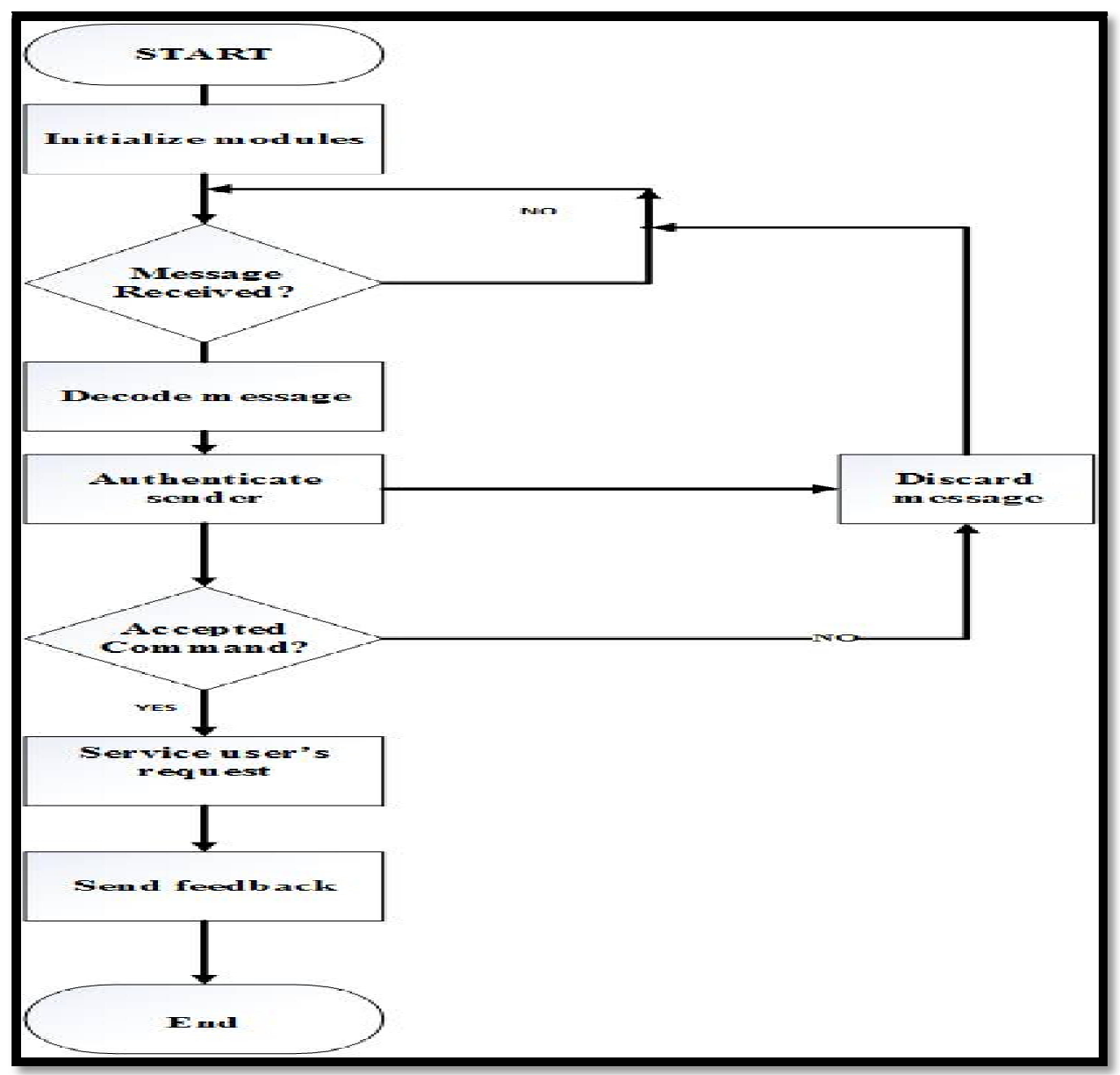

Figure 3: Flowchart 
After initializing the modules, the Arduino microcontroller monitors the GSM modules to receive user's request. The module uses the RS232 serial communication protocol. However, the GSM module requires the AT command set to carry out specific function. The following are some of the major AT commands used in this work. AT+CMGR=1 reads message number $1, \mathrm{AT}+\mathrm{CMGD}=1$ delete the $\mathrm{SMS}$, $\mathrm{AT}+\mathrm{CSMS}$ selects message service. On receiving a message, the content of the message is copied, verified for user authenticity and accurate control sequence. If the message is from an unauthorized user or not well formatted, it is discarded and no action is taken by the processing unit. A well formatted message received from an authorized user is decoded to determine the user's request.

The researchers used GSM wireless communication for controlling home appliances. We send some commands like "\#Alight on*", "\#A.light off*" and so on for controlling AC home appliances. After receiving given commands by Arduino through GSM, Arduino send signal to relays, to switch ON or OFF the home appliances using a relay driver. Here we have used a prefix in command string that is "\#A.". This prefix is used to identify that the main command is coming next to it and * at the end of string indicates that message has been ended. When we send SMS to GSM module by Mobile, then GSM receives that SMS and sends it to Arduino. Now Arduino reads this SMS and extract main command from the received string and stores in a variable. After this, Arduino compare this string with predefined string. If match occurred, then Arduino sends signal to relay via relay driver for turning ON and OFF the home appliances. And relative result also prints on 16x2 LCD by using appropriate commands as shown in Table 4.3.1.

\subsection{System Specification}

\subsubsection{Hardware Requirements}

- The Arduino UNO (Microcontroller)

- The GSM Shield (The Phone module)

- Liquid Crystal Display (LCD for display status)

- 4 channel Relay Module (For switching from primary circuit to secondary circuit

- Battery (power source)

- Electrical appliance (Bulbs as demonstration)

- Mobile phones

\subsubsection{Software Requirement}

- Arduino IDE

- Proteus Stimulator

- GSM network for sms messaging

\subsubsection{Functional Requirements}

The functional requirement of this project is to control electrical appliances using sms.

\subsubsection{Non-Functional Requirements}

- $\quad$ Reporting the status of electrical appliances.

- Limiting other phone contact from access the system

\section{System Design and Implementation}

\subsection{Introduction}

This chapter discusses the actual system design, we show you how the users interact with the system and show some diagrams of the user interfaces, the chapter also shows the packaging of the system and system schematics. The chapter will also describe how the system implementation should be done.

\subsection{System Design}

Systems design is the process of defining the architecture, modules, interfaces, and data for a system to satisfy specified requirements. Systems design could be seen as the application of systems theory to product development. So, in this subchapter, we show how the system was designed and assembled.

\subsubsection{Process Design}

Process design can be defined using two separate words in its definition. Design: "To design" refers to the process of originating and Process design can be defined using two separate words in its definition. Design: "To design" refers to the process of originating and developing a plan for a product, service or process. Process: Is any part of an organization which takes a set of input resources which are then used to transform something into outputs of products or services 


\subsubsection{Sequence Diagram}

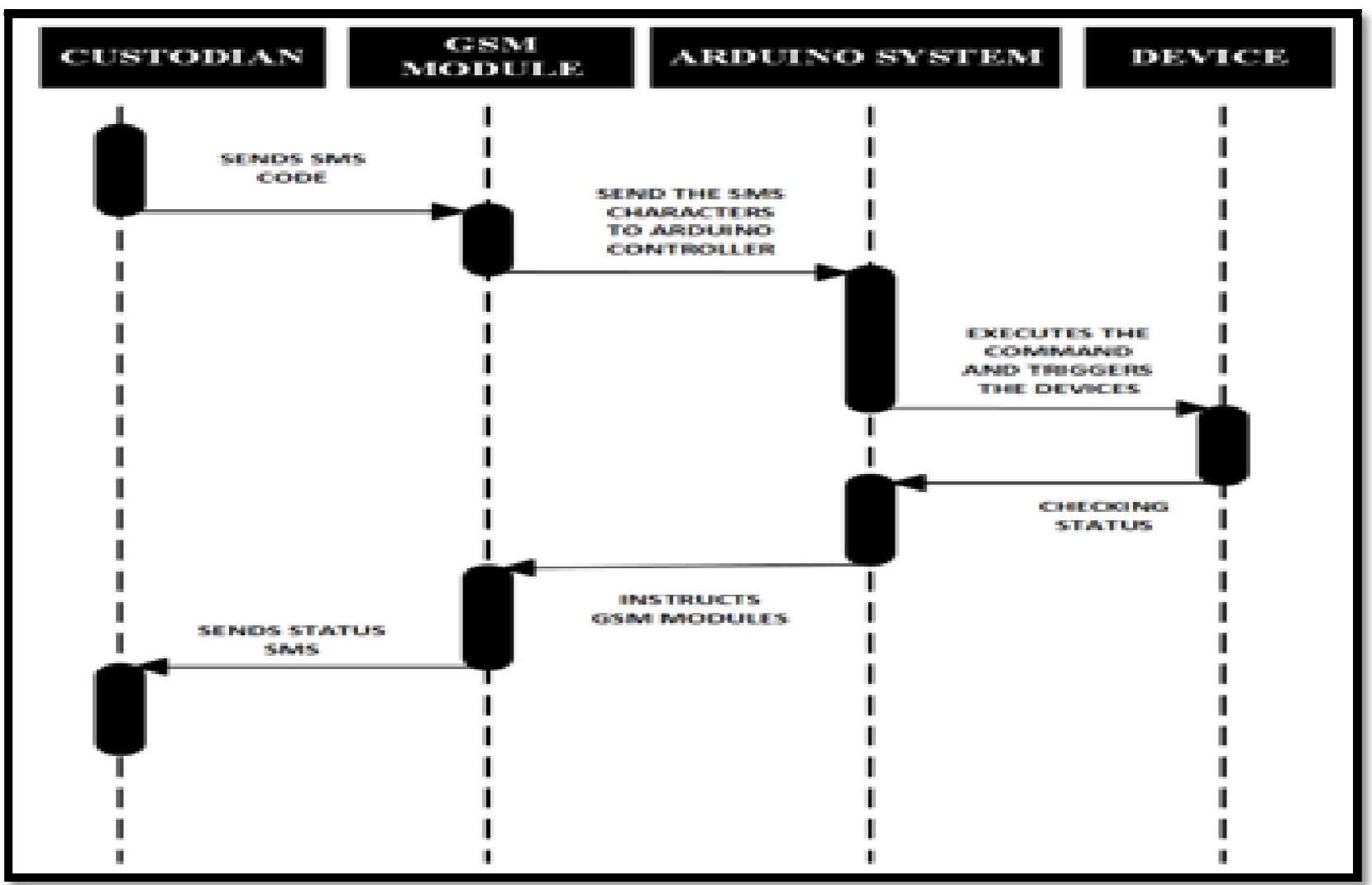

Figure 4: Sequence Diagram of the System

4.2.2.1. Use Case Diagram

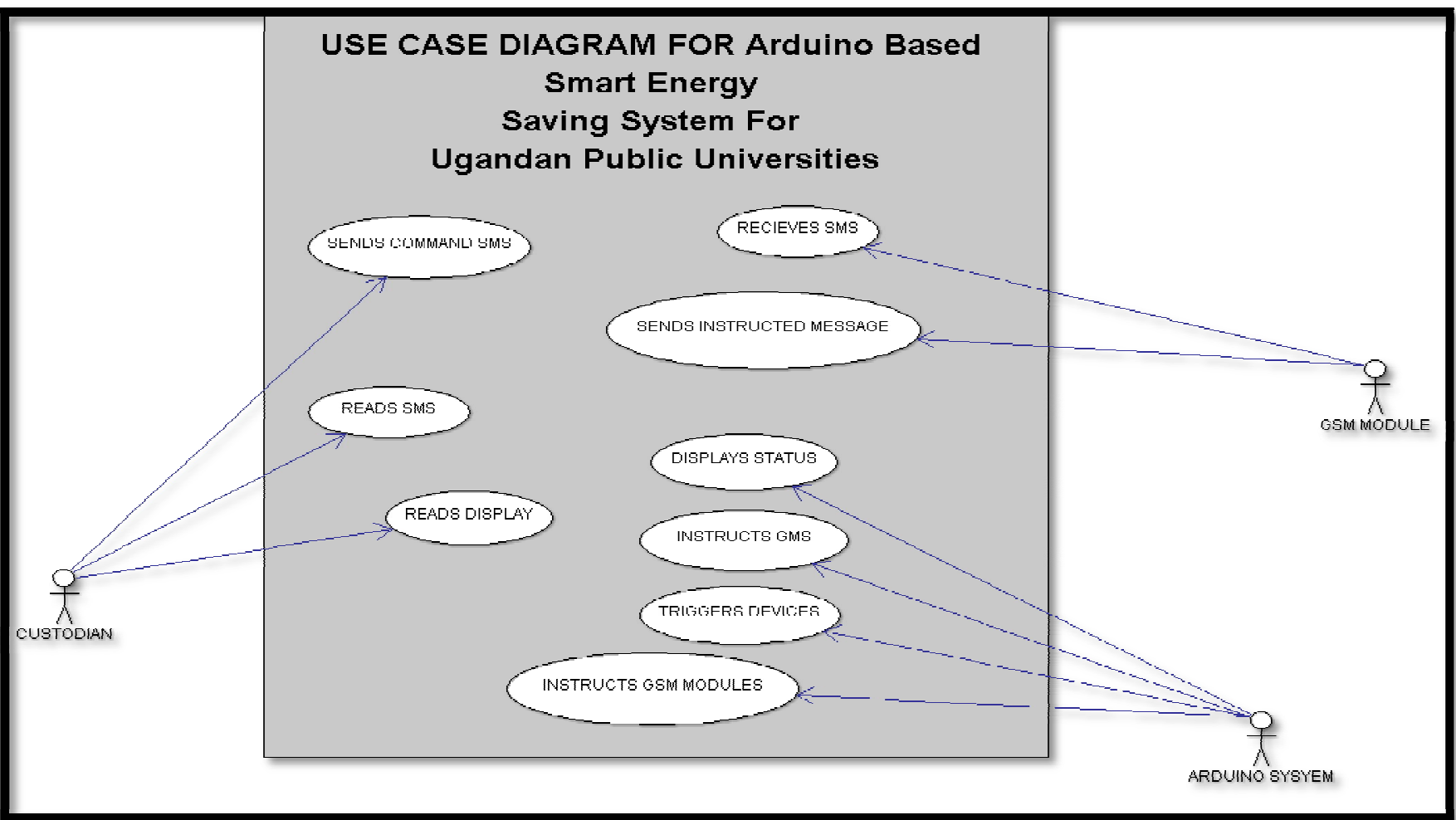

Figure 5: Use Case Diagram of the system 


\subsubsection{State Diagram}

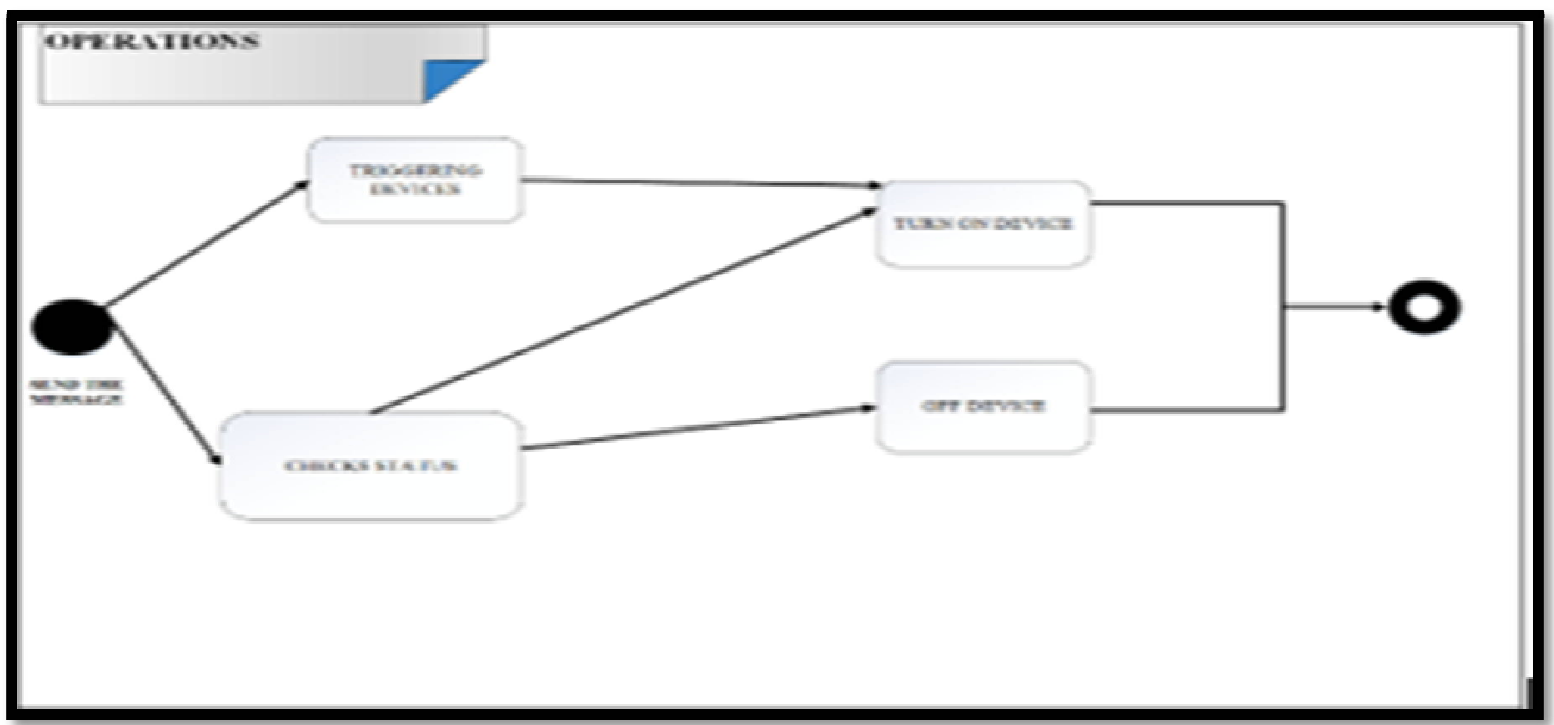

Figure 6: State Diagram of the System

\subsubsection{Schematic design}

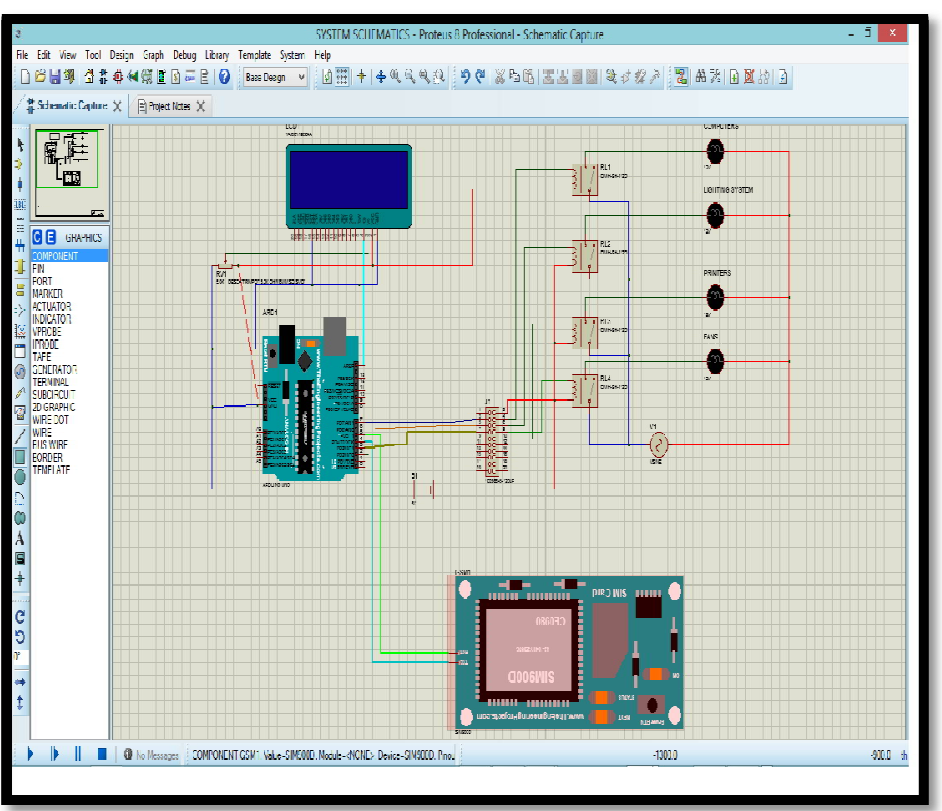

Figure 7 : The Schematics Layout of the System Interface Design

4.2.1.5. System Interface on Smart Phone

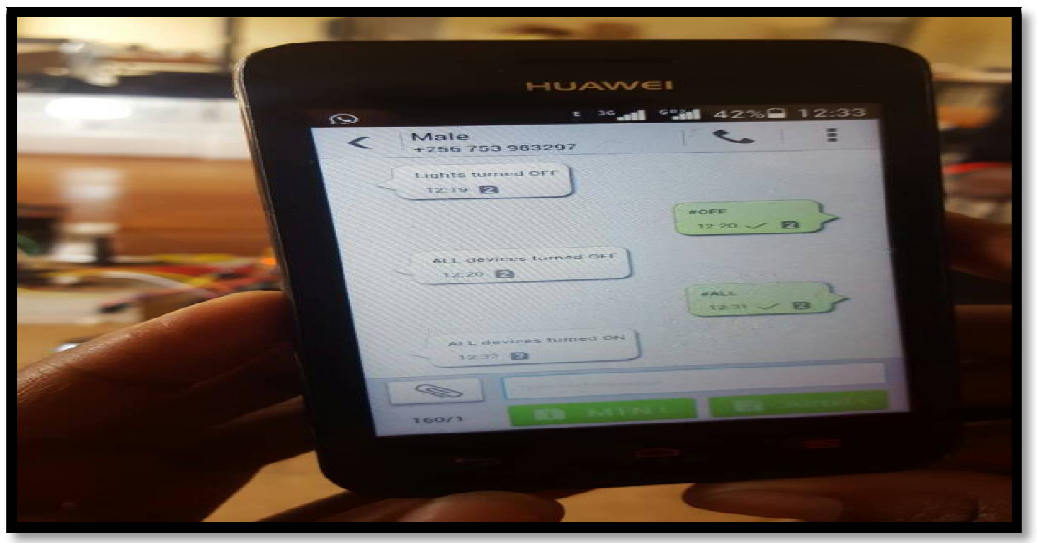

Figure 8: Interface of the Systems Normal SMS Interface 


\subsection{System Implementation}

This chapter focuses on implementation of smart energy saving system in muni university setting. The focus is put on the main functionality of the system such as sending and receiving of the sms that triggers the electronic devices. The system needs to undergo several development processes so as it can be readily available and used. The following are the necessary steps that need to be followed in order to fully implement the energy saving system in the university.

\subsubsection{Conversion}

We would consider to use pilot conversion which is the process of converting from the old system by installing the entire new system but using it only in part of the organization. We would take a single block as a pilot for example we try out the system on the library block and after the concerned personnel are conversant with the system, it is then installed on the other blocks of the organization. We considered it for its advantages which include;

- There is no need for additional personnel.

- It allows pro-training of personnel with the new system.

- It causes minimum destruction/ disruption unlike the direct change over method.

- These methods are usually less costly.

- The risks associated with errors or failures are limited.

- It allows the system to be implemented quickly.

\subsubsection{System Deployment}

During the installation, the system requires accessories including electric cables, contactors among others. The deployment diagram is as show below.

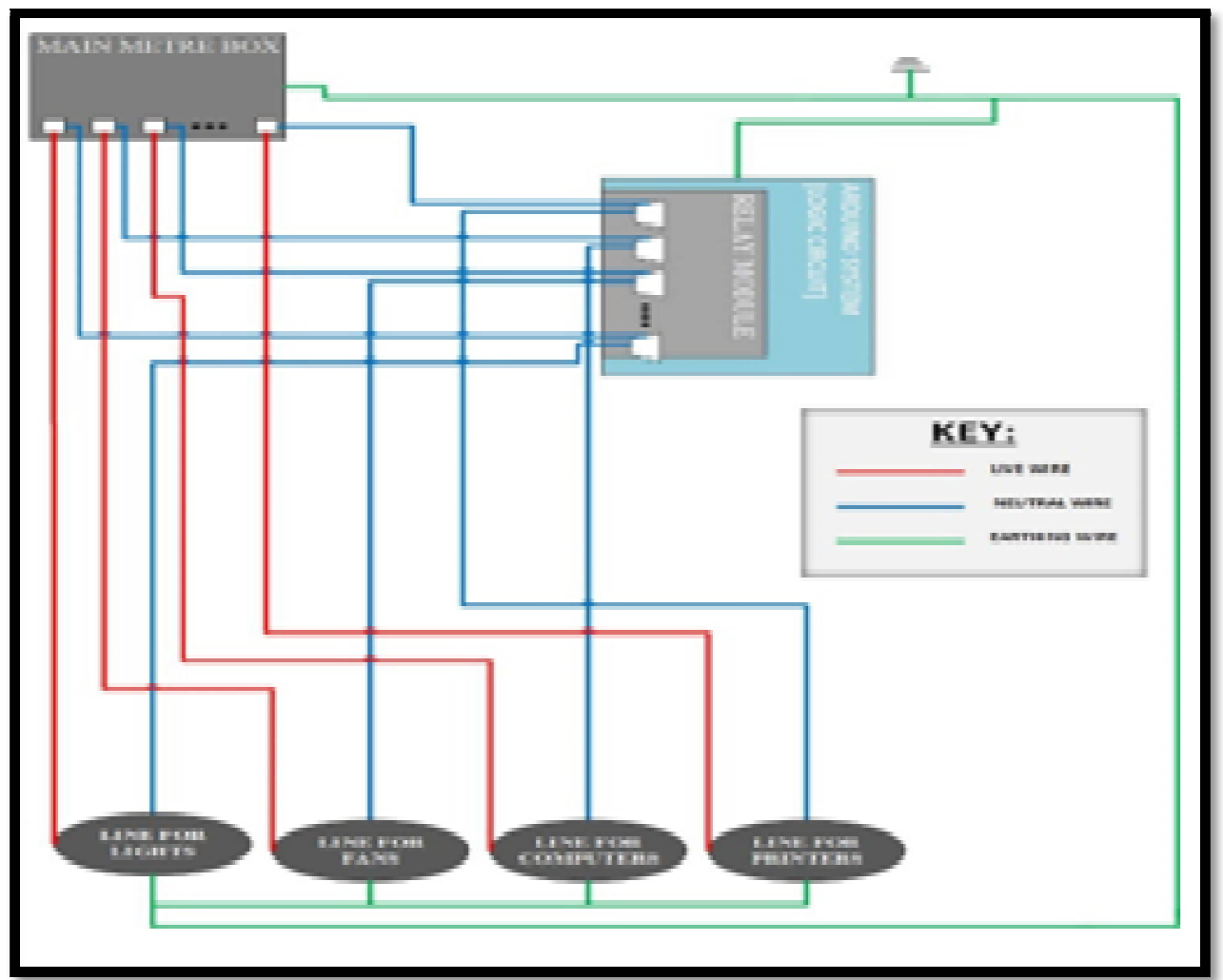

Figure 9: The Installation Block Diagram for System

\subsubsection{Final Assembled Project Prototype}

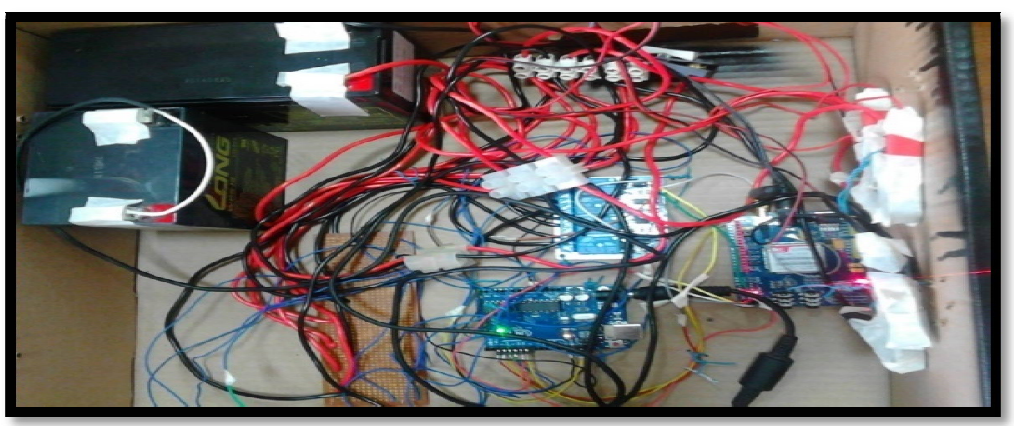

Figure 10: Project's Prototype Internal Assembly 


\subsubsection{Final Project Package}

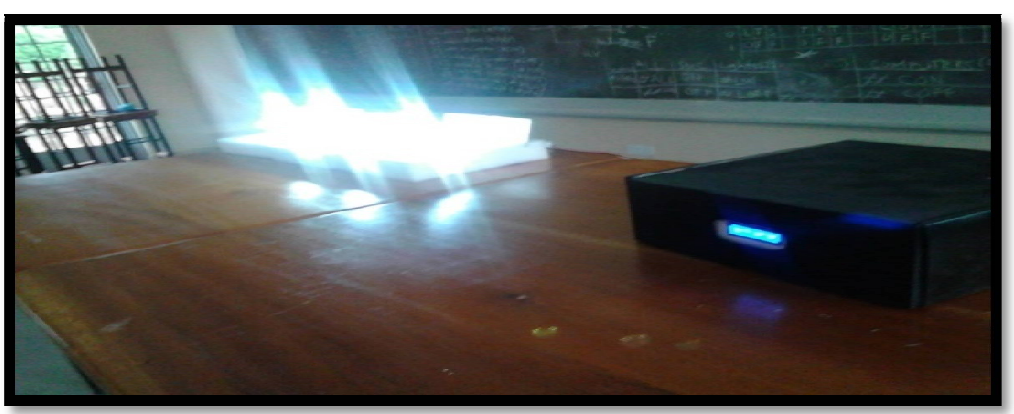

Figure 11: Final Project Package

\begin{tabular}{|c|c|c|}
\hline Appliances & Switching On & Switching Off \\
\hline Lights & Lon & Loff \\
\hline Computers & Con & Off \\
\hline Fans & All & \\
\hline All & All & \\
\hline Checking Status & Status & \\
\hline
\end{tabular}

Table 2: System's Operation

\section{Ethical Consideration and Limitations}

\subsection{Introduction}

We were ethical conscience as we performed our research about this project. However, our ethics was elevated by the good will from our participants and stakeholders' involvement. Using the guidelines as explain in this section, we did not harm or violate any of our participants.

\subsection{Technical, Deployment and Other Considerations}

\subsubsection{Environment Friendly}

This system as you have seen it was designed and implemented as reported to save energy, thus the amount of toxic produced by functioning electrical appliances when there are on is reduced instantly as soon as they are turned off automatically. This also reduces the occurrence of global warming.

\subsubsection{Fairness and Equity}

While collecting user requirements for the system we did not discriminate any gender, region, literacy-level or disability. We picked all the details and organized them in a right way irrespective of the region, technology, and other attributes that were given by the Estates Engineers, University Staff and the Students at large.

\subsubsection{Ethical Consideration}

As professionals we preserve the integrity of data about individuals. And for this reason, the data collected from the different respondents will not be disclosed to anybody.

\subsubsection{Unauthorized Access}

Estate engineer office will access the system resources after deployment. Once the system is released and deployed, the developers shall be compelled not to access the system unless permitted by the client(s).

\subsubsection{Quality}

The proposed system interface is user friendly, fast and easy to use for all the stakeholders due to its great level of convenience while using SMS from mobile phone. The system has been de

\section{Conclusion and Recommendation}

\subsection{Introduction}

We now summaries the findings, the recommendations for future improvements and lessons learnt from this project.

\subsection{Conclusions}

The completion of this project has almost fulfilled the objectives of developing an embedded system and designing an energy saving system for Ugandan Public Universities using Arduino. The whole project demonstrated the idea of developing the system that could reduce the electricity consumption by proper monitoring of the unnecessary power usage and notifying the custodian in time. The system has a wide range of application since its GSM based. There is no such particular boundary or specialized area for implementation of the project. The system can be implemented in homes, 
offices and organizations. The team members have completed the project with best abilities to perform smoothly and also with the further enhancements by which one could definitely increase the potential of the system. Throughout the project a framework has been developed that uses hardware and software in developing a low cost, powerful, accurate and reliable smart energy saving using Arduino different components. Arduino Uno, relay module and GSM module are used that provides higher performance with low power consumption. It has smooth functionality that provides the expected output. The system has been tested and found out to be accurate and reliable. Through the process of system analysis to its evaluation, different challenges were faced and overcome fulfilling the objectives of the project. This project proved to be very beneficial for us in all aspects. The project provided us with an opportunity of working in different areas of engineering, both hardware and software. Teamwork has been the major factor without which this project would not have been possible. The project was successfully developed meeting the objectives of the project and gaining lots of experience in the related field which will contribute to the academic and professional skills.

\title{
6.3. Recommendations
}

One of our recommendations is that during the development of our system we were not able to include the functionality were by when the university is able to use the system using Wi-Fi and it will need no money to buy airtime, so we recommend using the Wi-Fi shield in a campus proximity. This system is recommended for every homes, offices, laboratories, hospitals and industries to aid those working or living in those places when it comes to controlling their appliances and equipment. This smart energy saving system has to be perceived by the schools and the world at large as a necessary and vital technological upgrade While working on this design, at some point in time we were carried away by the fact that our project is working as desired but on further thinking, we thought about the fact that our control system should working independently on its own when it comes to the power supply unit, that the system get its power from the public power supply system which is well known that it is not stable, so on this note, we will advise any further work on this control system to have its power supply independent on its own or better still use rechargeable batteries or solar if possible. We also recommend in case the organization have a private network like Wi-Fi the system can be switched from using sms to using the data from the private network and this can be done through using the Wi-Fi shield

\section{References}

i. Greichen, J. J. (1992, August). Value based home automation on today's market. IEEE Transaction on Consumer Electronics,

\author{
i. Vol.38, pp.34-38.
}

ii. Afshan, M., Jay pal, B., \& Amol, B. (2014). DTMF Based Automation System with Reduction of Noise Using Goertzel DFT Estimation. Fourth International Conference on Communication Systems and Network Technologies (CSNT), 1124-1129. doi:10.1109/ CSNT.2014.230

iii. Alheraish, A. (2004, Novemeber). Design and implementation of home automation system. IEEE Transactions on Consumer Electronics (Volume: 50, Issue: 4, Nov. 2004), Vol.50 (No.4), 1087-1092. doi:10.1109/ TCE.2004.1362503

iv. Alkar, A. Z., \& Buhur, U. (2005). IEEE Transactions on Consumer Electronics, Vol.62(No.4), 1169-1174. doi:10.1109/ TCE.2005.1561840

v. Amponsah, P, F., Gifty Addo-Danquah, R., Korkoi Bonku, L., \& Saro-Adu, K. (2018). Investigating Unconventional Abbreviations in Sms Texts. International Journal of Applied Linguistics and English Literature. 7. 25. 10.7575/ aiac.jjalel. v.7n.4p.25.

vi. Anwaarullah, S., \& Altaf, S. v. (2013). RTOS based Home Automation System using Android. International Journal of Advanced Trends in Computer Science and Engineering, Vol.2 (No.1), pages 480-484. Retrieved September 27, 2017,

from

https:/ / s3.amazonaws.com/ academia.edu.documents/ 32301503/ icacsesp87.pdf?AWSAccessKeyId=AKIAIWOWY YGZ2Y53UL3A\&Expires=1507588717\&Signature=Gyf4ekK1Uyw22njivIRQI9JxMZo\%3D\&response-contentdisposition=inline\%3B\%20filename\%3DRTOS based Home Automation System u

vii. Bala Krishna, Y., \& Nagendram, S. (2012). Zigbee Based voice control system for smart home. International Journal of Computer Technology \& Application, Vol.3 (No.1), 163-168. Retrieved sept 30, 2017, from https:/ / scholar.google.com/ scholar?hl=en\&as sdt=0\%2C5\&q=Y.+B.+Krishnatand+S. +Nagendram $\% 2 \mathrm{C}+\% \mathrm{E} 2 \% 80$ \%9CZigbee+based +voice+control+system+for+smart+home\%2C\% E2\%80\%9D+Int.+J.+Comput.+Technol.+Appl. $\% 2 \mathrm{C}+$ vol.+3\%2C+no.+1\%2C+pp.+163\%E2\%80\%93168\%2C+2012.\&btnG=

viii. Baraka, K., Ghobril, M., Malek, S., Kanji, R., \& Kayssi, A. (2013). Low Cost Arduino/ Android-Based Energy-Efficient Home Automation System with Smart Task Scheduling. Fifth International Conference on Computational Intelligence, Communication Systems and Networks (CICSyN), 2013, 296-301. doi:10.1109/ CICSYN.2013.47

ix. Carelin, F., \& Raglend, J. I. (2011, July). Home automation using GSM. International Conference on Signal Processing, Communication, Computing and Networking Technologies (ICSCCN), 2011, 15-19. doi:10.1109/ ICSCCN.2011.6024506

x. Coyne, I. T. (1997). Sampling in qualitative research. Purposeful and theoretical sampling; merging or clear boundaries? Journal of advanced nursing, 26(3), 623-630.

xi. E Source Companies LLC) (2003). Managing Energy Costs in Colleges and Universities.

xii. https:/ / www9.nationalgridus.com/ non html/ shared_energyeff_college.pdfManaging Energy Costs in Colleges and Universities. https:// www9.nationalgridus.com/ non_h̄tml/ shared_energyeff_college.pdf 
xiii. Jieming, Z., Xuecai, G., Yucang, Y., Hang, L., Zhati, A., \& Xiaoyan, C. (2010, October 2nd). Developing a voice control system for Zigbee-based home. IEEE International Conference Network Infrastructure and Digital Content, 737731. doi:10.1109/ ICNIDC.2010.5657880

xiv. Jinsoo, H., Yun, J., \& Park, K.-r. (2010). User-friendly home automation based on 3D virtual world. IEEE Transactions on Consumer Electronics, Vol.56(No.3), 1843-1847. doi:10.1109/ TCE.2010.5606335

xv. John, R., Dilek, B., \& Ziya, A. A. (2010). P based home automation system. IEEE Trans. Consum. Electron, Vol.56(No.4), 2201. doi:10.1109/ TCE.2010.5681091

xvi. Kanagamalligg, S., Vasuki, D., Priya, V., \& Viji, V. (2014, May). A Zigbee and Embedded Based Security Monitoring and Control System. International Journal of Information Sciences and Techniques, Vol.4(No.3), 177-178. RetrievedSeptember2017, from https:/ / scholar.google.nl/ scholar?hl=nl\&as_sdt=0\%2C5\&q=S.+Kanagamalliga\%2C+S.+Vasuki\%2C+A.+V.+Priya\% 2C+and+V.+Viji\%2C+\%E2\% 80\%9CA+Zigbee+and+Embedded+based+Security+Monitoringtand+Control+System $\% 2 \mathrm{C} \% \mathrm{E} 2 \% 80 \% 9 \mathrm{D}+$ Int.+J.+Inf.+Sci.+Tech.\%2C+vol.+4\%2C+no.+3\%2C+pp.

xvii. Khusvinder, G., Shuang-Hua, Y., Yao, F., \& Xin, L. (2009, May). A zigbee-based home automation system. IEEE Transactions on Automation Science and Engineering, Vol.55 (No.2), 167-168. Retrieved September 27, 2017, from http:/ / ieeexplore.ieee.org/ abstract/ document/ 5174403/

xviii. Manohar, S., \& Kuman, M. (2015). E-mail Interactive Home Automation System. Int. J. Comput. Sci. Mob. Comput., Vol.51(No.4), 78-87. Retrieved Oct 10, 2017

xix. Mohd, A. S., \& Veda, M. C. (2013). Design of Remote Intelligent Smart Home System Based on Ziggbee and GSM Technology. International Journal of Engineering Trends and Technology, Vol.4 (No.9), 3926-3929. Retrieved September 26, 2017, from https:/ / scholar.google.com/ scholar?hl=en\&as sdt=0\%2C5\&q=M.+A.+Samadtand+Mv.+CHARY\%2C+\%E2\%80\%9 CDesign+of+Remote+Intelligent+Smart+Home-System+Based+on+Zigbee+and+GSM+Technology $\% 2 \mathrm{C} \% \mathrm{E} 2 \% 80$ \%9D+Int.+J.+Eng.+Trends+Technol.+\%28IJETT\%29\%E2\% 80\%93Volume\%2C+vol.+4\%2

xx. Motshed, N. M., Muid-Ur-Rahman, G. M., Karim, R. M., \& Zaman, U. H. (2015). Microcontroller based home automation system using Bluetooth, GSM, Wi-Fi and DTMF. International Conference on Advances in Electrical Engineering, 101-104. doi:10.1109/ ICAEE.2015.7506806

xxi. Oladlmeji, S., Mustapha, H. B., \& Lukman, A. (2017). Development of a Low-Cost GSM-Bluetooth Home Automation System. International Journal Intelligent Systems and Applications, 41-50. doi:10.5815/ ijisa.2017.08.05

xxii. Piyare, R., \& Tazil, M. (2011). Bluetooth based home automation system using cell phone. International Journal Of Electronics Communication Engineering And Technology, 192-195.

xxiii. Prantosh, K., Paul, C., \& Dipak, C. (2017). Usability Experience Design, Cloud Computing and Green Computing:. Asian Journal of Information Science and Technology, Vol.6, 40-44. Retrieved Nov 4, 2027

xxiv. Shaker, M. N., \& Fahtha, A. A. (2009). Friendly home automation system using cell phone and J2ME with feedback instant voice messages. International Conference on Computer Systems and Applications, 2009. AICCSA 2009. IEEE/ ACS, 531-538. doi:10.1109/ AICCSA.2009.5069375

xxv. Siwen, L., \& Yunhong, L. (2004). Design and Implementation of Home Automation System. International Syposium on Information Science and Engineering, Vol.2 (No.4), 1087-192. doi: 10.1109/ ISISE.2008.90

xxvi. Sougata, D., Nilava, D., Rishabhh, D., Sayantan, D., \& Apurba, G. (2014). Embedded system for home automation using SMS. First International Conference on Automation, Control, Energy and Systems (ACES), 2014. doi:10.1109/ ACES.2014.6808026

xxvii. Statt, N. (2016, April 04). (Nest) Retrieved August 20, 2017, from THE VERGE: https:/ / www.theverge.com/ 2016/ 4/ 4/ 11362928/ google-nest-revolv-shutdown-smart-home-products

xxviii. [26] Tanvir, M. A., \& Partha, P. B. (2008). Home appliances control using mobile phone. International Conference on Advances in Electrical Engineering, Vol.54(No.2), 251-254. doi: 10.1109/ ICAEE.2015.7506843

xxix. Teymourzadeh, R., Salah, A. A., Chan, W. K., \& Hoong, M. V. (2013). Smart GSM based Home Automation System. IEEE Conference on Systems, Process \& Control (ICSPC), 306-309. doi: 10.1109/ SPC.2013.6735152

xxx. Tulijappa, M. L., Sanjay, M. L., Kaarthik, S. R., Alok, R., \& Nayan, D. (2009). Control of remote domestic system using DTMF. International Conference on Instrumentation, Communication, Information Technology, and Biomedical Engineering, 1-6. doi:10.1109/ ICICI-BME.2009.5417279

xxxi. UMEME. (2018). Electricity Retail Base Tariffs for 2018. Kampala: UMEME LIMTED. Retrieved March 25th, 2018, from https:/ / www.umeme.co.ug/ file/ Tariffs.pdf

xxxii. Vini, M., \& Reddy, S. R. (2012). GSM-Bluetooth based Remote Monitoring and Control System with Automatic Light Controller. International Journal of Computer Applications, Vol.46 (No.1), 20-28. Retrieved Oct 11, 2017

xxxiii. University World News. (2019). Will students be forced to pay the utility bills? https:/ / www.universityworldnews.com/ post.php?story=20160607123128658

xxxiv. Wahab, A., Mohd, H., Abdullah, Abdul, K., \& Herdawatie. (2010). GSM based electrical control system for smart home application. Journal of Convergence Information Technology, Vol.5 (No.1), 33-39. Retrieved September 30, 2017, from http:/ / eprints.uthm.edu.my/ 3058/

xxxv. Yan, M., \& Shi, H. (2013, February). Smart Living Using Bluetooth-Based Android Smartphone. International Journal of Wireless \&Mobile Networks, Vol. 5(No. 1), 8.

xxxvi. Zhao, Y., \& Zhaohui, Y. (2008). A low-cost GSM/ GPRS based wireless home security system. IEEE Transactions on Consumer Electronics, Vol.54(No.2), 567-572. doi:10.1109/ TCE.2008.4560131. 